\title{
VoICE AND DATA APPLICATIONS IN DIFFERENTIATED SERVICE INTRANETS
}

\author{
M. Angulo-Bernal ${ }^{1}$, M.A. Turrubiartes-Reynaga' ${ }^{1}$, D. L. Torres-Roman ${ }^{1} \&$ M.A. Argumedo-Ledon ${ }^{2}$
}

1Centro de Investigación Científica y de Estudios Avanzados, Unidad Guadalajara

Departamento de Ingeniería Eléctrica

Prolongación López Mateos Sur No. 590, C.P. 45090, Guadalajara, Jal.

Email:mangulo@gdl.cinvestav.mx,mturrubi@gdl.cinvestav.mx,dtorres@gdl.cinvestav.mx

2 Universidad Autónoma de Baja California

Facultad de Ingeniería

Blvd.. Benito Juárez s/n, Unidad Universitaria, C.P. 21900, Mexicali, B.C.

Email:mledon@info.rec.uabc.mx

Received: November $5^{\text {st }}, 2001$. Accepted: January $27^{\text {st }}, 2003$

\section{ABSTRACT}

One of the options that telephonic systems have, in order to optimize the required bandwidth, is to migrate to statistical multiplexing systems. These multiplexing schemes also allow the convergence with current data communications systems. This work is based on the interconnection of telephonic systems through the data network using digitalization and voice compression techniques. The optimization of the resources is achieved using the voice compression standard of $8 \mathrm{kbps}$ instead of the $64 \mathrm{kbps}$ standard, thus introducing differentiated services in IP networks. The performance of the voice packets is improved in terms of delay and packet losses. The results presented here obtained by means of computer simulations using COMNET. Real telephonic traffic information acquired for several months at the UABC was used. This allowed the consideration of effects such as: busy hours and variable call duration. The performance of the system was evaluated, obtaining very satisfactory results in terms of resources utilization.

\section{RESUMEN}

Una de las opciones del sistema telefónico para optimizar el ancho de banda requerido, es migrar a sistemas de multicanalización estadística, que permitan además la convergencia con los actuales sistemas de comunicación de datos. Este trabajo se basa en la interconexión de los sistemas telefónicos a través de la red de datos, empleando técnicas de digitalización y compresión de voz. La optimización de recursos se logra al utilizar el estándar de compresión de voz a 8kbps en lugar del estándar de 64kbps, de este modo al introducir servicios diferenciados en redes IP el desempeño de los paquetes de voz es mejorado en términos de retardo y pérdida de paquetes. Los resultados presentados fueron obtenidos mediante simulaciones en computadora empleando COMNET, en el cual se utilizó información de tráfico telefónico real obtenido de la tarificadora telefónica principal en la UABC, información procesada por varios meses, que permite considerar efectos tales como hora pico y duración variable de llamada.

El desempeño de los esquemas es evaluado, obteniendo resultados muy satisfactorios en términos de utilización de recursos.

KEYWORDS: Voice over IP networks, Differentiated Services, Quality of Service 


\section{INTRODUCTION}

The IP networks globalization and the increase in the diversity of applications and services demands that they become capable of managing different quality of service requirements, an example of Dynamic Bandwidth Allocation Schemes is presented in [1]. Unlike the voice network, data networks use information packets and packets switching devices to transmit the information, thus they optimize the usage of the information transmission capabilities. This paper is based on the interconnection of the telephonic systems by means of E\&M signaling through the data network, using digitalization and voice compression techniques and international standards.

This paper is organized as follows: the second section gives a brief introduction to the telephone system, in the third section Fundamental concepts of IP networks that offer differentiated services are described, the next section contents the simulation considerations, followed by the results presentation and analysis. Finally, the conclusions are presented.

\section{TELEPHONE SYSTEM}

The typical behavior of packed voice sources is as follows [2]. A source is said to be "active"when a user is literally speaking, during this period, the voice source generates packets of fixed length at regular time intervals. Afterwards, comes a silence period during which the source changes it state to "inactive" and no packets are produced. When we identify these two states of the process, it is possible to model the voice with Markovian chains since they allow us to characterize a discrete variable that changes in the time domain through its states transition probabilities.

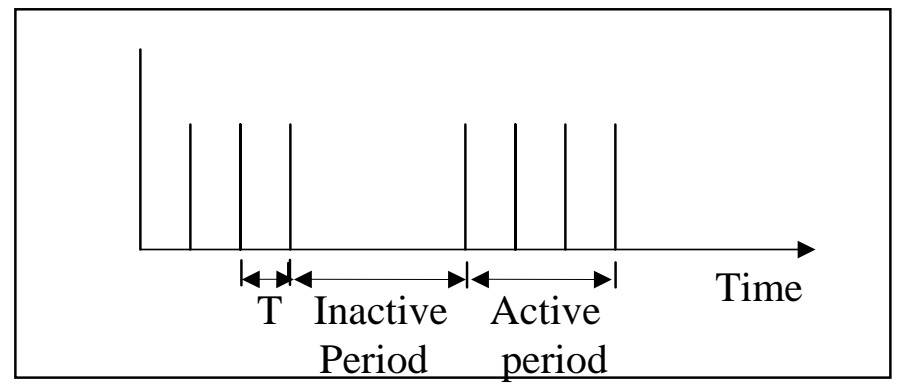

Figure 1. Packetized voice source

A packet burst from a voice source is being modeled by arrivals at fixed intervals with a Tmsecs duration, and by a silence period during which there is no arrival (figure 1).

According to Heffes [3], a voice source is modeled by the next equation:

$$
F(t)=\left[(1-\alpha T)+\alpha T\left(1-e^{-\beta(t-T)}\right)\right] U(t-T)
$$

Where $U(t)$ is the singled step function, is the average value of the active period, is the average value of the silence period; both periods are described by exponential distribution functions and $\mathrm{T}$ is the voice sample inter-arrival time during active periods.

\section{DIFFERENTIATED SERVICES}

Differentiated Services is an architecture that proposes scalability by means of aggregated traffic classification states. They are implemented in the network layer where the category of a packet is marked in one or more fields of the IP layer header. The differentiated services can be implemented under two modes: Expedited Forward and Assured Forward. 
The Expedited Forward mode was designed to support connections with low losses, delays and jitter. This mode shows a behavior similar to the virtual dedicated lines between two final points with a peak bandwidth.

The Assured Forward mode defines four related service classes, where each one supports three discharge priority levels. When congestion occurs in the router, the packets with lower priority are followed by those with higher priority. The four assured forward classes do not have the values of bandwidth and delay defined.

The architecture proposed by RFC 2475 [4], defines that the differentiated services must classify individual micro flows at the gate of the network in one of four classes of services. The admission to the network is based on the analysis of one or more fields in the packet. The packet is marked (changing some header bits of fields) to specify that it belongs to certain kind of service class, then, it is admitted in the network.

\section{SIMULATION CONSIDERATIONS}

In figure 2, we can observe a voice outline through IP network. This work does not consider the E\&M signaling process, focusing in the analysis of router and packetizer. The codification standards used are the G729A (CS-CELP) and the $\mathrm{G} 732[5,6]$.

In the packetizing process the delay of the process must be considered as well as the size of the generated packet, including the headers of the protocols required for the information transport. In the equation 2, the load due to one single voice source and a sample of the voice coder in the packet are represented.

$$
\text { Load }[b p s]=\left[M_{b}+E t_{b}+I P_{b}+U D P_{b}+R T P_{b}\right] /(M t)
$$

Where $\mathrm{Mb}$ represent the sample size expressed in bits, Etb, IPb, UDPb, RTPb represents the Ethernet, IP, UDP and RTP headers size(bits) respectively, and $\mathrm{Mt}$ represents the coder sample generation interval expressed in sec.

$$
\text { Load }[\text { bps }]=\left[n M_{b}+\left(E t_{b}+I P_{b}+U D P_{b}+R T P_{b}\right)\right] /(n M t)
$$

From equation 3, we can observe that the load of the network decreases as $n$ increases, where $n$ is the number of voice samples in a packet, however there are other parameters considered in this simulation such are packet losses, maximum delay and delay variations. Besides, it is necessary to take into account that a big part of the IP network traffic is not voice, but data, so data traffic such as www represents outbursts and increases the stowage in the interconnection devices.

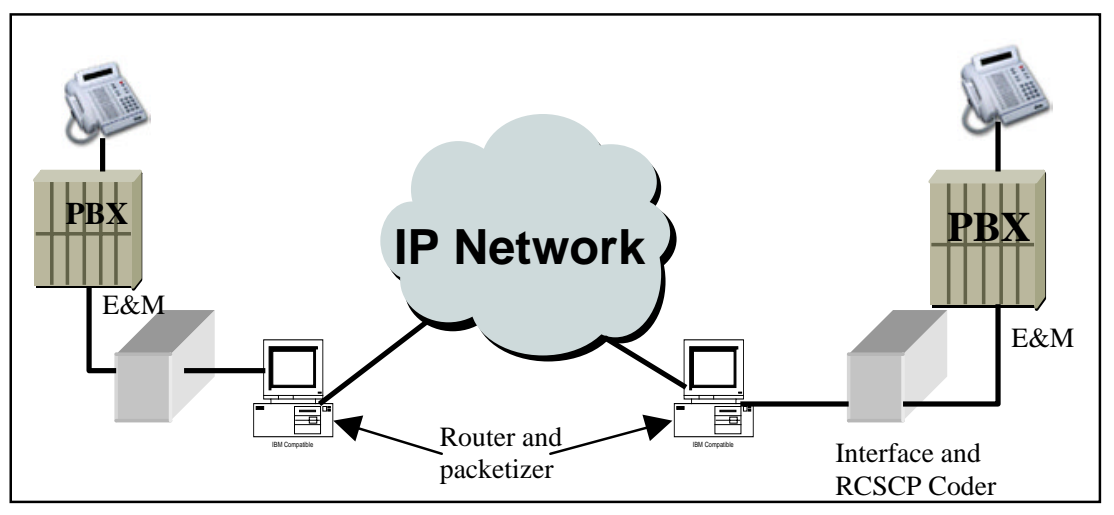

Figure 2. Implemented scheme of voice over IP 
Differentiated Services are used to give priority to voice packets over data packets inside the routers; discarding the usage of upper layer protocols such are RTP/ RTCP.

The IP network was simulated using COMNET (see figure 3), where the call generators, background traffic generators, coders, packetizers and routers were simulated. The IP network service the packets depending of their service priority, where the voice packets service rate is twice the remaining packets service rate. The primary function of an IP router is to direct the packet received through an input port to an output port depending on the destiny address of the packet and the routing table. To simulate the effects all the traffic converges in the same output port.

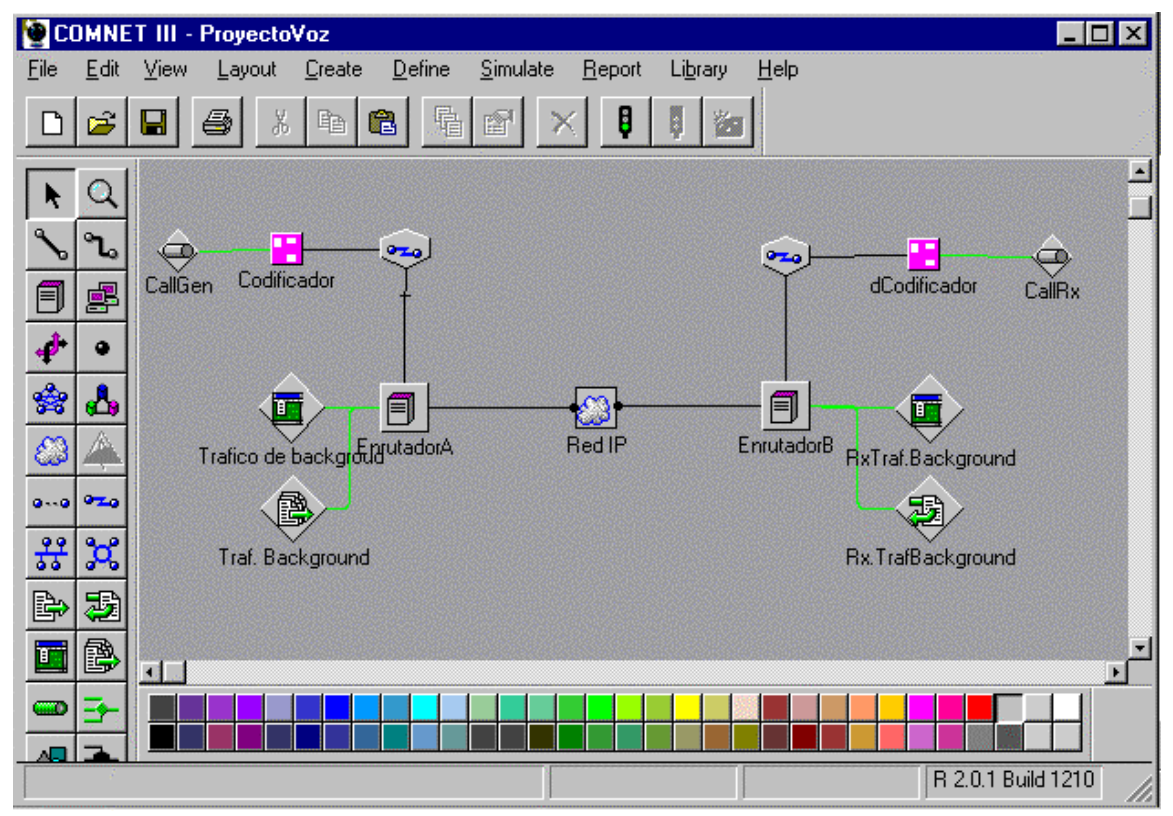

Figure 3. Voice over IP model, implemented in COMNET

The parameters evaluated in the simulation were the delay, voice packet losses and the usage of the routers links. Although the main goal of this work was the optimization of the bandwidth in the trunk links, it was verified that the outline did not degrade one of the most significant parameters of the voice service quality: the delay. Figure 4 shows us a graphic of the obtained delay in voice packets with an $8 \mathrm{kbps}$ codification. The voice packet losses average during 500 seconds of simulation was $0.7 \%$, which is below the $1 \%$ limit. The used outline with an 8 kbps codification shows an effective reduction of $30 \%$ of the bandwidth in the transmission line respect to $64 \mathrm{kbps}$.

Figure 5 is a photo of the testing circuit of the RCSCP voice coder circuit (from Conexant Semiconductor Systems), where with help of a logic state analyzer its performance was verified.

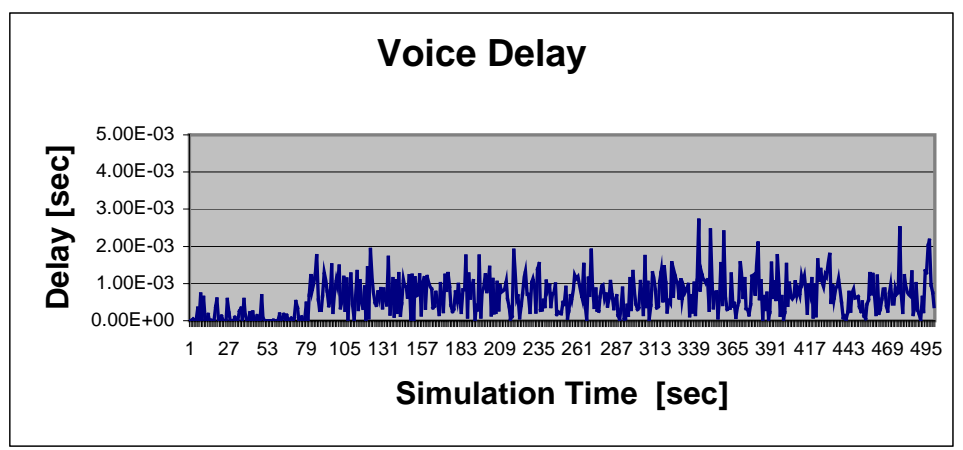

Figure 4. Voice delay obtained by simulation with skbps compression 


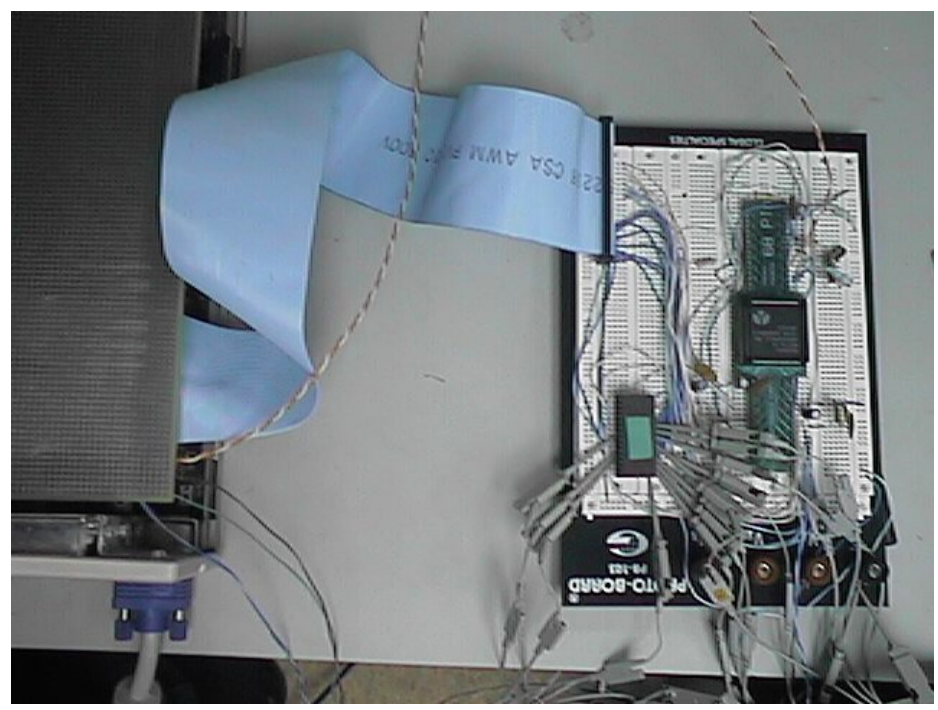

Figure 5.- Voice coder circuit under test

\section{CONCLUSIONS}

The performance evaluation of the outline used was made doing an effective reduction of $30 \%$ in the bandwidth of the transmission link. The result was very satisfactory in terms of resource utilization, an acceptable voice performance was maintained, the voice packet losses average during simulation was $0.7 \%$, which is below the $1 \%$ limit, and the average packet delay was $0.645 \mathrm{msec}$.

As a future work, it is considered the implementation in a printed circuit of the prototype showed in the testing circuit, and to characterize the system as well as introduce in the packetizer the necessary software to handle RTP/ RTCP protocols.

\section{ACKNOWLEDGEMENTS}

To the Autonomous University of Baja California through the Engineering Faculty and to DGIP for their support to the project "Voice over IP networks, study case: UABC". The work of the first and second author was supported by a PROMEP scholarship.

\section{REFERENCES}

[1] Angulo, M., Gallardo, J., Makrakis, D. Dynamic Bandwidth Allocation Schemes for a Differentiated-ServiceCapable Internet Boundary Node. International Conference on Telecommunication, Acapulco México, 2000.

[2] Daigle, John N., Langford, Joseph D., Models for Analysis of Packet Voice communications systems . IEEE Journal on selected areas in communications VOL SAC-4 No.6 p 847-854. 1986.

[3] Heffes, Harry, Lucantoni, David., A markov Modulated Characterization of Packetized Voice and Data Traffic and Related Statistical Multiplexer Performance. IEEE Journal on selected areas in communications Vol SAC-4 No.6 pp 856-867. 1986.

[4] Blake, S., Black, D., Carlson, M. An architecture for Differentiated Services. Internet Engineering Task Force, RFC 2475. 1998.

[5] ITU Reg. G.729, Coding speech at 8 kbps using Conjugate-Structure Jalgebraic-Code-Excited Linear Prediction CS-ACELP. 1996.

[6] ITU Reg G.729a, Reduced Complexity 8kbps CS-ACELP Speech Codec, 1996. 
Authors Biography

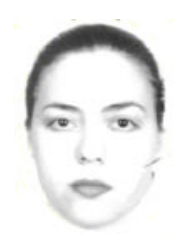

Marlenne Angulo

Received the M.Sc. degree from the Department of Electronic and Telecommunications CICESE research center in Ensenada, Mexico. Currently, she is pursuing the Ph.D. degree at the CINVESTAV Research Center, Guadalajara Mexico. Her research interests include Quality of Service and multimedia.

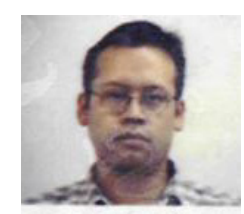

Marco Turrubiartes

Received the M.Sc. degree from the Department of Electronic and Telecommunications CICESE research center in Ensenada, Mexico, and studied the bachelor in communications and electrical engineering at UANL(Autonomous University of Nuevo Leon). Currently, he is pursuing the Ph.D. degree at the CINVESTAV Research Center, Guadalajara, Mexico. He is an IEEE member. His research interests include Quality of Service and characterization of Internet Traffic.

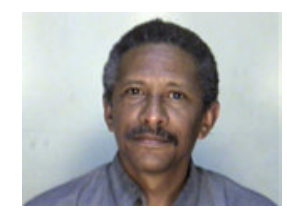

\section{Deni Torres-Roman}

Received a Ph.D. degree in telecommunications from Technical University Dresden, Germany in 1986. He was a professor at the University of Oriente, Cuba. Coauthor of a book about Data Transmission. He received the Telecommunication Research Prize in 1993 from AHCIET Association and was recipient of the 1995 Best Paper Award from AHCIET Review, Spain. Since 1996 he is an associate professor at Research and Advanced Studies Center of IPN (Cinvestav-Mexico). His research interests include VLSI, hard and software designs for telecommunication area. He is a member of the IEEE.

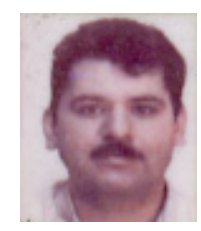

\section{Marco Antonio Argumedo Ledón}

He studied Electrical \& Mechanic Engineer specialized in electronic and Telecommunication Specialization at Universidad Autónoma de Baja California, México (Autonomous University of Baja California). Currently he works as Technical management and support in Telecommunications for General Department of Informatics at UABC and also is a teacher in Engineering Faculty at UABC. He has worked in the development of packet networks. 